Farum

Sociológico

\section{Forum Sociológico}

Série II

24 | 2014

Circulação de saberes e desafios em saúde

\title{
Desafíos metodológicos de la investigación cualitativa iberoamericana en salud. En la confluencia de tres vertientes
}

Methodological challenges for Ibero-American qualitative health research. At the confluence of three streams

\section{Carolina Martínez-Salgado}

\section{OpenEdition \\ Journals}

\section{Edición electrónica}

URL: https://journals.openedition.org/sociologico/1041

DOI: 10.4000/sociologico.1041

ISSN: 2182-7427

Editor

CICS.NOVA - Centro Interdisciplinar de Ciências Sociais da Universidade Nova de Lisboa

Edición impresa

Paginación: 47-53

ISSN: 0872-8380

Referencia electrónica

Carolina Martínez-Salgado, «Desafíos metodológicos de la investigación cualitativa iberoamericana en salud. En la confluencia de tres vertientes», Forum Sociológico [En línea], 24 | 2014, Puesto en línea el 01 noviembre 2014, consultado el 31 marzo 2022. URL: http://journals.openedition.org/sociologico/ 1041 ; DOI: https://doi.org/10.4000/sociologico.1041

Este documento fue generado automáticamente el 31 marzo 2022.

(c) CICS.NOVA 


\title{
Desafíos metodológicos de la investigación cualitativa iberoamericana en salud. En la confluencia de tres vertientes ${ }^{1}$
}

\author{
Methodological challenges for Ibero-American qualitative health research. At \\ the confluence of three streams
}

Carolina Martínez-Salgado

\section{Introducción}

1 La evocación de los principales desafíos metodológicos que desde mi propia experiencia con esta modalidad de indagación en el ámbito de la salud podría identificar, me llevó casi de inmediato a preguntarme hasta dónde mis percepciones serían compartidas por otros investigadores que trabajan con este tipo de aproximación. Así que me di a la tarea de revisar lo que se ha escrito al respecto en los últimos años, primero en publicaciones en español y portugués, y luego también en otros idiomas, principalmente en inglés (esta suerte de idioma-puente que nos hace posible asomar a algo de lo que ocurre en otros mundos distintos al que nos es accesible a través de nuestra propia lengua). Inicialmente, mi revisión se centró en el ámbito específico de la investigación cualitativa en salud, que es el que reune a nuestra comunidad, pero como aparecieron bajo mi lente varias reflexiones sugerentes, procedentes de la investigación cualitativa en otras áreas, no quise dejar de incorporar a algunas de ellas.

Mi búsqueda, como en realidad suelen ser todas las búsquedas, fue bastante personal. Porque por muy sistemática que al principio intenté ser, de inmediato empezaron a surgir, en el camino, múltiples destellos invitadores para extender la exploración en las más diversas direcciones. Y, como suele hacerse en investigación cualitativa, no quise renunciar a perseguir, en la medida de lo posible, esas pistas. Así, cuando llegó a su fin 
el tiempo disponible - porque, como también lo saben quienes tienen experiencia en investigacion cualitativa, el material a indagar es siempre mucho más abundante de lo que podemos abarcar y difícilmente se agota -, me percaté de que me sería imposible reportar exactamente cuál fue el camino recorrido para recolectar las piezas a partir de las cuales tracé el panorama que aquí voy a mostrar².

\section{Primeras impresiones}

De lo que sí puedo dar cuenta es que la primera y muy general impresión que se fue forjando en mi mente a lo largo de esta revisión fue que los investigadores cualitativos en salud enfrentamos desafíos metodológicos que compartimos con quienes emprenden esta modalidad de indagación en cualquier otro ámbito de la vida de los humanos, pero que nuestros desafíos toman, además, otras configuraciones muy particulares, impuestas por la peculiar materia de la que nos ocupamos. Un ejemplo, tomado de mi propia experiencia, es el hecho de que la problemática de salud, cualquiera que sea el ángulo desde el que la abordemos, se remite de un modo $u$ otro a situaciones que involucran a nuestro ser encarnado, nuestra corporeidad simbolizada, y nos coloca ante las diversas amenazas (reales o fantaseadas) que se ciernen sobre ella, con las múltiples y complejas implicaciones que esto supone.

Una segunda impresión, surgida también en esa primera etapa de mi revisión, fue que hay algunas especificidades en las preocupaciones sentidas por los investigadores cualitativos de habla hispana y portuguesa ${ }^{3}$ distintas a las que manifiestan otras comunidades lingüísticas, y también algunas diferencias entre quienes se ocupan de la salud y los que trabajan en otros temas. De esta suerte, quienes escribimos en español y portugués hemos comentado nuestra desagradable sensación ante la aparente invisibilidad (o falta de escucha) a la que suele enfrentarse toda comunicación no escrita en inglés, a pesar del gran peso cuantitativo que tiene en el mundo la población (y los investigadores) de habla española y portuguesa ${ }^{4}$.

5 Por lo que se refiere a las inquietudes más específicas de los investigadores cualitativos iberoamericanos que trabajamos sobre el tema de la salud, a lo anterior podemos añadir la percepción que todos tenemos del papel hegemónico que tiene, en nuestro campo, la investigación biomédica, con el experimento como estándar de oro, y la perspectiva epidemiológica dominante que no concede valor a ningún conocimiento que no involucre la medición. De hecho, en aquellas situaciones en las que llega a prevalecer un statu quo científico acríticamente subordinado a los dictados de la visión más reduccionista de la ciencia, lo anterior puede llevar a exclusiones poco compatibles con el verdadero espíritu científico, y más cercanas a lo dogmático, casi inquisitorial.

De manera que, hasta ese momento, podría haber agrupado a los desafíos metodológicos en los que mi propia experiencia y lo que iba apareciendo en esta búsqueda en cuatro grandes conjuntos, según se tratara de la preocupación de:

7 a) los investigadores cualitativos en general (en todo el mundo y sobre las diversas temáticas),

b) los investigadores cualitativos en salud (en todo el mundo),

c) los investigadores cualitativos iberoamericanos (que se ocupan de diversas temáticas), $\mathrm{y}$

d) los investigadores cualitativos iberoamericanos que se ocupan del tema de la salud. 
11 Sin embargo, llegada a ese punto, decidí concentrar mi atención en la investigación cualitativa en salud con especial énfasis en la iberoamericana, aunque sin dejar fuera las inquietudes expresadas por quienes se ocupan de la salud en otras partes del mundo y publican en inglés (lo cual los hizo accesibles a mi lectura). Al hacerlo, surgió la tercera de las impresiones que quiero aquí reportar, la cual finalmente definió el sentido que elegí para organizar los desafíos que me pareció identificar, en tres distintas vertientes, si bien todas ellas entretejidas entre sí de la manera más intrincada. Las tres vertientes que me pareció percibir fueron estas:

12 1. Los desafíos que plantea la existencia misma de este tipo de abordaje de la realidad, y la necesidad de mostrar el valor de sus aportaciones, en un contexto en el que reina soberana la así llamada medicina basada en la evidencia, con su propensión a adueñarse de toda la legitimidad y el reconocimiento.

13 2. Los desafíos que brotan de la vertiente que voy a designar como epistémicometodológica, íntimamente relacionados con los anteriores, ya que son los que proporciona los cimientos a la solidez y coherencia en las que se funda su legitimidad.

14 3. En forma simultánea con las dos anteriores, los desafíos que denominaré prácticoéticos, que se desprenden no sólo de la ejecución de toda investigación cualitativa, sino además de la materia de la que con ella nos ocupamos: la problemática de salud.

En lo que sigue me detendré a considerar brevemente los retos que ubicaría en cada una de estas vertientes.

\section{Primera vertiente: el desafío de la existencia misma}

16 Me parece que esta modalidad de aproximación al conocimiento de la realidad, en el ámbito específico de las ciencias de la salud, enfrenta de entrada un primer desafío que es el de hacerse escuchar, entender, reconocer y respetar, con toda la complejidad de su sofisticada epistemología, en un medio en el que imperan de manera hegemónica otras creencias sobre lo que es el mundo, lo que podemos conocer de él y la forma en que resulta aceptable estudiarlo; otra ontología, otra epistemología y, por tanto, otra metodología (De la Cuesta, 2006; Smythe \& Giddings, 2007; Jiménez, 2011). Aún cuando las ciencias de la salud se ubican justamente en la encrucijada de las ciencias naturales y las ciencias sociales, como es sabido, en la formación de la mayor parte de nuestras profesiones predomina el conocimiento científico natural. La convicción que ahí prevalece es que la cientificidad está dada por la posibilidad de medición de los fenómenos, bajo los supuestos de neutralidad y objetividad. Desde esa visión, la investigación cualitativa puede ser descalificada por subjetiva y, como tal, anecdótica, poco confiable y muy distante de la solidez que se atribuye a las así llamadas "ciencias duras" (Mercado et al., 2011; Jiménez, 2011; Neergaard et al., 2009).

De esta suerte, no es fácil transmitir la idea de que, en realidad, ocurre todo lo contrario. Porque para algunas de las tradiciones filosóficas que dan sustento a ciertas modalidades de investigación cualitativa, lo que aparece como epistemológicamente ingenuo es, justamente, la pretensión de ignorar el papel del intérprete, que es nada menos que el sujeto que habla a través de la voz de la ciencia (Kincheloe, 2005). Pero este es, de todos modos, un primer y nada despreciable desafío, que podemos colocar dentro del grupo más amplio de los retos de la comunicación, de la transmisión del valor, fundamentos, implicaciones y posibilidades de esta forma de aproximación a la 
realidad (Bosi, 2012). Y en este punto la escritura como modo de transmisión no es un problema menor (Blanco, 2011; Martínez, 2010; Wolcott, 2004).

En esta vertiente podría colocar también el especial relieve que toman, en el terreno de la investigación cualitativa en salud, las preocupaciones relacionadas con el rigor y la calidad (Cádiz, 2006; Castillo \& Vásquez, 2003, entre muchos más) ${ }^{5}$, sobre las cuales han trabajado con mucho tino, varios de nuestros colegas (Bosi, 2012; Conde, 2002; Calderón, 2002).

A este grupo de inquietudes hay que añadir la de buscar soluciones epistemológicamente coherentes a la polémica sobre la conmensurabilidad o inconmensurabilidad de las aproximaciones cuantitativas y las cualitativas, de la cual varios de nosotros nos hemos sentido en la necesidad de ocuparnos (Popay, 2003; Martínez, 2008) ${ }^{6}$.

Pero estos no son más que algunos ejemplos del tipo de desafíos que me parecen provenir de esta primera vertiente, los cuales se conectan de manera bastante inmediata con los que vienen de la segunda, que enuncio a continuación.

\section{Segunda vertiente: desafíos epistémico- metodológicos}

21 Los desafíos que se nos presentan desde esta segunda vertiente son tantos, que se requerirían una exposición mucho más amplia que la que aquí ofrezco, con el mero señalamiento de algunos ejemplos de aquello a lo que en este punto quiero aludir.

Para comenzar, están los desafíos que derivan de la necesidad de asumir los numerosos y difíciles aspectos inherentes a estas formas de acercamiento a la realidad, como son el problema de la intersubjetividad, en el vínculo en vivo con los participantes en cada estudio, y la intertextualidad, en los vínculos diferidos que se producen entre cada uno de los textos en los que dejamos constancia de nuestras versiones, y cada lector con el que, a través de ellos, interactuamos. Dimensiones íntimamente relacionadas, por cierto, con la reflexividad a la que mencionaré un poco más adelante.

Está también el problema que plantea nuestra condición de investigadores encarnados, con todos los significantes implicados en nuestro ser corporal (nuestra edad, género, etnia, complexión, estatura, condición de salud, enfermedad o discapacidad), tanto en lo que se refiere a lo que nuestra presencia puede significar para aquellos a quienes convocamos a participar en cada uno de nuestros estudios (Sharma et al., 2009; Brown y Boardman, 2011), como los significados que ellos, en su ser de carne y hueso, evocan en nosotros (Martínez, 2009; Kai et al., 2007).

24 Y no podemos dejar de mencionar el perpetuo desafío de la interpretación (Minayo, 2012; Hunt, 2009; Crist \& Tanner, 2003), que nos obliga a reconocer las muy diversas y complejas identidades histórica, social y culturalmente moldeadas de cada uno de los participantes en un estudio cualitativo, en tanto que en esta forma de trabajo las fronteras entre quien plantea la indagación y aquellos a quienes se refiere no son ya nunca más claras (Dickson-Swift et al., 2006).

Un desafío más es el que plantea el compromiso de ser consecuentes con el tipo de conocimientos que creemos posible generar. Una de las más clásicas objeciones a la investigación cualitativa es la de cómo generalizar. Las respuestas a esta pregunta se 
encuentran en el reconocimiento de que el conocimiento es siempre producido por alguien situado en un contexto (De la Cuesta, 2006), y que también el conocimiento tiene una historia.

Por si con todo esto no bastara, está la aspiración de desarrollar nuevas epistemologías, de encontrar nuevas formas de generar conocimiento más acordes con la diversidad de perspectivas desde las que se percibe la complejidad de los problemas que deseamos estudiar, una tarea en la cual, según se aprecia en la bibliografía publicada, se encuentra especialmente comprometido el enfoque de la enfermería en algunas de sus combinaciones con las visiones feministas (Aranda, 2006).

Muy lejos de haber esbozado de manera completa todos los desafíos que se plantean en esta vertiente que por ahora he nombrado como epistémico-metodológica, entraré ahora a la tercera para ocuparme, aún si es también sólo a manera de un apunte, de este tercer conjunto de desafíos tan vinculados con los dos previamente mencionados.

\section{Tercera vertiente: desafíos práctico-éticos}

Esta tercera vertiente es, a mi juicio, la que adquiere las mayores particularidades en el terreno específico de la salud. De ella emergen un conjunto de desafíos que asumen características muy propias cuando se trata de aspectos con tanta trascendencia - a veces inmediata - para la experiencia humana, el sufrimiento y la vida misma, como son todos los que se refieren a la problemática de salud (Wilson, 2011). Si para tratar adecuadamente la segunda vertiente se hubieran requerido muchas páginas más, para desarrollar con alguna suficiencia esta tercera se requeriría, seguramente, al menos un libro entero. Pero asomemos a ella aunque sea por un momento.

Entre los desafíos metodológicos que aquí podemos encontrar hay que mencionar, de entrada, el espinoso tema de la asimetría inherente a la condición de ser el afectado o el que estudia al afectado por un problema de salud (Carter et al., 2008).

Junto con esto, aparece el delicado asunto de las relaciones de poder, que se relacionan con situaciones como la aceptación o no para participar en el estudio, las diversas formas de hacerlo, o de resistirse a ello pese a todos los esfuerzos persuasivos por parte del investigador, hasta los momentos porlos que pasa la relación en todas y cada una de las etapas del proceso de investigación mismo (Martínez, 1999; Nunkoosing, 2005; Karnieli-Miller et al., 2009). Como lo reconocen Aléx y Hammarström (2008), este sería, justamente, uno de los componentes involucrados en el concepto de reflexividad; en efecto, como ellas lo muestran en sus ejemplos, en cada situación de entrevista se crean, a través del discurso y otros dispositivos, ciertos juegos, complejos y sutiles, en las relaciones de poder entre entrevistado y entrevistador, que requieren ser tomados en cuenta.

31 Están también las cuestiones referentes a la veracidad de la información aportada que, como bien se ha sugerido, habría que pensar más bien en términos de la autenticidad de la versión que finalmente ofreceremos. Como lo discute Nunkoosing (2005), en investigación cualitativa hay que tener claro que la verdad no es algo fijo, incluso ni siquiera algo conocido, para nuestros informantes. La verdad, en cambio, parece ser algo que se va produciendo en la relación dialógica que, en cada momento nuevo de la experiencia, suele ir cambiando. Se trata, pues, de algo siempre en construcción, en 
remodelación. Una vez más, aquí, todo indica que la ingenuidad epistemológica y metodológica está en creer lo contrario (Kincheloe, 2005).

Un desafío más a considerar en esta vertiente es el que plantea el trabajo con temas sensibles, y la especial capacitación que su manejo requiere por parte del investigador, no sólo por las difíciles emociones que esta práctica suscita en él, sino también y de manera muy importante para lidiar en forma adecuada y no dañina con las intensas emociones que emergerán en los participantes en el estudio (Martínez, 2009; Malacrida, 2007; Dickson-Swift et al., 2006; Johnson \& Clarke, 2003).

Otro punto problemático especialmente desafiante es el que surge ante la gran interrogante de qué es ético, y cómo podemos saberlo, con toda la incertidumbre que esta pregunta conlleva, en especial cuando se trata de orientar nuestras acciones en la práctica cotidiana, ya sea como profesionales de la salud, como investigadores o en ambos lugares (Ramos et al., 2010; Peñaranda et al., 2011; Nunes, 2008; Hewitt, 2007; Doane \& Varcoe, 2008; Doane et al., 2009; Hunt, 2009; Millet et al., 2008; Goldblatt et al., 2011).

Por último, y no porque no haya muchísimo más que decir al respecto, quiero mencionar el reto que nos plantea responder con veracidad a la importante pregunta de cuáles son los intereses a los que servimos, nos percatemos de ello o no - y ojalá intentáramos hacerlo - con la elaboración de cada uno de nuestros estudios.

\section{En la confluencia de tres vertientes: ¿un nudo borromeo o un nudo gordiano?}

Para terminar este breve relato de mi experiencia ante la pregunta por los desafíos que enfrentamos hoy día los investigadores cualitativos iberoamericanos en salud, y esta suerte de inventario preliminar en el que he intentado registrar algunas de mis impresiones, quiero hacer explícito que, como de seguro será ya evidente, los elementos que proceden de cada una de las vertientes que traté de identificar no pueden separarse unos de otros. En efecto, conforme avanzaba en su reconocimiento y trataba de dar forma a esta comunicación, me fui dando cuenta de que quizá lo que me llevó a tratar de construir esta imagen fue la necesidad de encontrar algún modo de organizar lo que se iba configurando. Pero al final de todo el ejercicio, tal parece que, si intentara representar visualmente la confluencia de estas tres vertientes, más que un nudo borromeo, que fue la primera imagen que evoqué para tratar de hacerlo, en realidad a lo que más podría asemejarse es a un nudo gordiano (Figura 1). Pero mi principal intención con este rápido recorrido no es más que la de evidenciar la multiplicidad de ángulos a los que hemos de atender, para mantener abierta nuestra atenta reflexión y alimentar el constante debate que cada uno de ellos merece.

Figura 1

En la confluencia de tres vertientes: ¿nudo borromeo o nudo gordiano? 

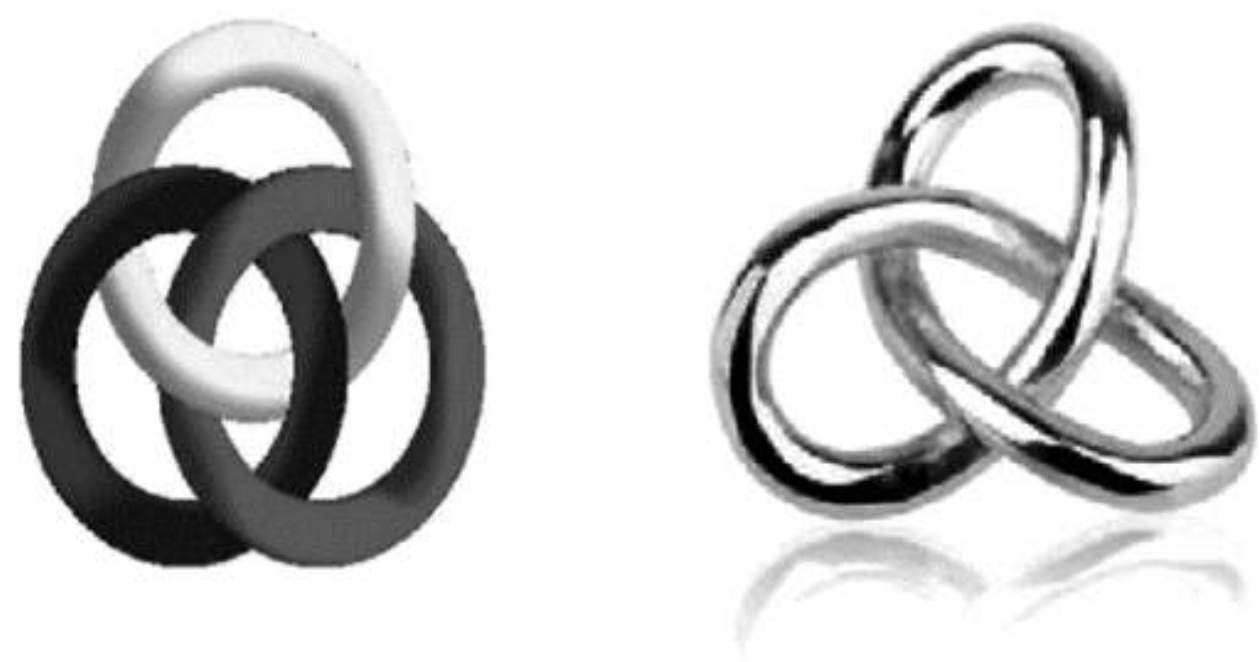

\section{BIBLIOGRAFÍA}

ALÉX, L. y A. Hammarström (2008), "Shift in power during an interview situation: methodological reflections inspired by Foucault and Bourdieu”, Nursing Inquiry, 15 (2), pp. 169-76.

ARANDA, K. (2006), "Postmodern feminist perspectives and nursing research: a passionately interested form of inquiry”, Nursing, 13 (2), pp. 135-143.

BLANCO, M. (2011), “Investigación narrativa: una forma de generación de conocimientos", Argumentos, 24 (67), pp. 135-156.

BOSI, M. L. M. (2012), “Pesquisa qualitativa em saúde coletiva: panorama e desafios”, Ciência \& Saúde Coletiva, 17c (3), pp. 575-586.

BROWN, L. y F. K. Boardman (2011), “Accessing the field: Disability and the research process”, Social Science \& Medicine, 72 (1), pp. 23-30.

CÁDIZ, J. (2006), “Rigurosidad científica y principios orientadores para el evaluador/ investigador", Ars Médica, 9 (9). Disponible en URL: http://escuela.med.puc.cl/publ/arsmedica/ arsmedica9/ars3.html (citado el 18 de agosto de 2012).

CALDERÓN, C. (2002), “Criterios de calidad en la investigación cualitativa en salud (ICS): apuntes para un debate necesario”, Revista Española de Salud Pública, 76 (5), pp. 473-482.

CARTER, S. M., C. F. Jordens, C. McGrath y M. Little (2008), "You have to make something of all that rubbish, do you? An empirical investigation of the social process of qualitative research", Qualitative Health Research, 18 (9), pp. 1264-76.

CASTILLO, E. (2011), “Lo científico de la investigación cualitativa. Viejos dilemas, nuevas posturas”, Nómadas, 18 (5), pp. 46-53. 
CASTILLO, E. y M. Vásquez (2003), “El rigor metodológico en la investigación cualitativa”, Colombia Médica, 34, pp. 164-167.

CASTRO, R. y M. Bronfman (1999), "Problemas no resueltos en la integración de métodos cualitativos y cuantitativos en la investigación social en salud", in M. Bronfmann y R. Castro (coords.), Salud, cambio social y Política. Perspectivas desde América Latina, México, EDAMEXInstituto Nacional de Salud Pública, pp. 49-64.

CONDE, F. (2002), "Encuentros y desencuentros entre la perspectiva cualitativa y la cuantitativa en la historia de la medicina”, Revista Española de Salud Pública, 76(5), pp. 395-408.

CONDE, F. y C. Pérez (1995), "La investigación cualitativa en Salud Pública", Revista Española de Salud Pública, 69, pp. 145-149.

CORNEJO, M. y N. Salas (2011), "Rigor y calidad metodológicos: un reto a la investigación social cualitativa”, Perspectivas. Individuo y sociedad, 10 (2). Disponible en URL: http:// www.psicoperspectivas.cl/index.php/psicoperspectivas/article/view/144/174 (citado el 19 de septiembre de 2012).

CRIST, J. D. y C. A. Tanner (2003), "Interpretation/analysis methods in hermeneutic interpretive phenomenology”, Nursing Research, 52 (3), pp. 202-205.

DE LA CUESTA, C. (2006), "Desafíos de la investigación cualitativa”, NURE Investigación 20. Disponible en URL: http://www.nureinvestigacion.es/ (citado el 12 de septiembre de 2012).

DICKSON-SWIFT, V., E. L. James, S. Kippen y P. Liamputtong (2006), "Blurring boundaries in qualitative health research on sensitive topics”, Qualitative Health Research, 16 (6), pp. 853-71.

DOANE, H. y C. Varcoe (2008), "Knowledge translation in everyday nursing: from evidence-based to inquiry-based practice”, ANS: Advances in Nursing Science, 31(4), pp. 283-95.

DOANE, H., J. Storch y B. Pauly (2009), “Ethical nursing practice: inquiry-in-action”, Nursing Inquiry, 16 (3), pp. 232-240.

GOLDBLATT, H., O. Karnieli-Miller y M. Neumann (2011), “Sharing qualitative research findings with participants: study experiences of methodological and ethical dilemmas", Patient Education and Counseling, 82 (3), pp. 389-95.

HEWITT, J. (2007), "Ethical components of researcher researched relationships in qualitative interviewing”, Qualitative Health Research, 17 (8), pp. 1149-59.

HUNT, M. R. (2009), "Strengths and challenges in the use of interpretive description: reflections arising from a study of the moral experience of health professionals in humanitarian work", Qualitative Health Research, 19(9), pp. 1284-1292.

JIMÉNEZ, M. E. (2011), “Desafíos de la investigación cualitativa en salud”, Gaceta Médica Boliviana, 34 (2), pp. 105-106.

JOHNSON, B. y J. M. Clarke (2003), “Collecting sensitive data: the impact on researchers”, Qualitative Health Research, 13 (3), pp. 421-34.

KAI, J., J. Bevan, C. Faull, L. Dodson, P. Gill y A. Beighton (2007), "Professional uncertainty and disempowerment responding to ethnic diversity in health care: a qualitative study", PLoS Medicine, 4 (11), pp. 1766-1775.

KARNIELI-MILLER, O., R. Strier y L. Pessach (2009), "Power relations in qualitative research", Qualitative Health Research, 19 (2), pp. 279-289. 
KINCHELOE, J. (2005), “On to the next level: Continuing the conceptualization of the bricolage", Qualitative Inquiry, 11 (3), pp. 325-350.

MALACRIDA, C. (2007), "Reflexive journaling on emotional research topics: ethical issues for team researchers", Qualitative Health Research, 17 (10), pp. 1329-39.

MARTÍNEZ, C. (1999), "Unexpected findings of a female team in Xochimilco, Mexico", Qualitative Health Research, 9 (1), pp. 11-25.

MARTÍNEZ, C. (2008), “Epidemiología e investigación cualitativa: tres posturas sobre las posibilidades de su enlace", Ponencia al III Congreso Iberoamericano de Investigación Cualitativa en Salud, Puerto Rico, San Juan, mayo de 2008.

MARTÍNEZ, C. (2009), “Qualitative inquiry with women in poverty in Mexico City. Reflections on the emotional responses of a research team", International Journal of Qualitative Studies in Education, 22 (2), pp. 297-313.

MARTÍNEZ, C. (ed.) (2010), Por los caminos de la investigación cualitativa. Exploraciones narrativas y reflexiones en el ámbito de la salud, Colección: Académicos de CBS, México, División de Ciencias Biológicas y de la Salud, Universidad Autónoma Metropolitana-Xochimilco.

MERCADO, F., B. Díaz, L. M. Tejada y C. D. Ascencio (2011), "Investigación cualitativa en salud: una revision crítica de la producción bibliográfica en México”, Salud Púbica de México, 53 (6), pp. 504-512.

MILLER, F. A., M. Giacomini, C. Ahern, J. S. Robert y S. de Laat (2008), "When research seems like clinical care: a qualitative study of the communication of individual cancer genetic research results", BMC Medical Ethics, 22 (9), p. 4.

MINAYO, M. C. S. (1993), "Quantitativo-Qualitativo: Oposição ou Complementariedade”, Cadernos de Saúde Pública, 9 (3), pp. 239-262.

MINAYO, M. C. S. (2012), “Análise qualitativa: teoria, passos e fidedignidade”, Ciência \& Saúde Coletiva, 17 (3), pp. 621-626.

NEERGAARD, M. A., F. Olesen, R. S. Andersen y J. Sondergaard (2009), "Qualitative description the poor cousin of health reseach?", BMC Medical Research Methodology, 16 (9), p. 52. Disponible en URL: http://www.biomedcentral.com/1471-2288/9/52 (citado el 23 de septiembre de 2012).

NUNES, E. D. (2008), "Ethical aspects considered by researchers who use qualitative approaches in health", Ciência \& Saúde Coletiva, 13 (2), pp. 351-360.

NUNKOOSING, K. (2005), “The problems with interviews", Qualitative Health Research, 15(5), pp. 698-706.

PEÑARANDA, F., C. Velez, G. Molina, E. Montoya y L. Bloom (2011), “La investigación cualitativa desde la reflexión ética: Un requisito para promover la justicia social”, Ponencia presentada en el Seventh International Congress of Qualitative Inquiry, University of Illinois at Urbana-Champain Illini Union, Urbana, IL, 17 de mayo de 2011.

POPAY, J. (2003), "Qualitative research ando the epidemiological imagination: a vital relationship”, Gaceta Sanitaria /S.E.S.P.A.S, 17 (supl. 3), pp. 58-63.

RAMOS, F. R. S., M. Finkler, E. R. Goncalves y J. C. Caetano (2010), “A eticidade na pesquisa qualitative em saúde: o dito e o não dito nas produções científicas", Ciência \& Saúde Coletiva, 15 (Supl. 1), pp. 1673-1684. 
SHARMA, S., S. Reimer-Kirkham y M. Cochrane (2009), "Practicing the awareness of embodiment in qualitative health research: methodological reflections", Qualitative Health Research, 19 (11), pp. 1642-50.

SMYTHE, L. y L. Giddings (2007), "From experience to definition: addressing the question «what is qualitative research?»”, Nursing Praxis in New Zealand, 23 (1), pp. 37-57.

WILSON, B. (2011), "The value of reflexivity in resolving ethical dilemmas research in care homes", Journal of Advanced Nursing, 67 (9), pp. 2068-77.

WOLCOTT, H. (2004), “Mejorar la escritura de la investigación cualitativa”, Investigación y Educación en Enfermería, 22 (2), pp. 150-162.

\section{NOTAS}

1. Comunicação apresentada no V Congresso Ibero-Americano de Pesquisa Qualitativa em Saúde - Circulação de Saberes e Desafios em Saúde, realizado em Lisboa entre 11 e 13 de Outubro de 2012.

2. Mi búsqueda inició en Redalyc, SciELO, LILACS y PubMed, para revisar lo publicado a partir del año 2000 que emergiero a partir de la combinación de las palabras clave "investigación cualitativa” y "desafíos" (en español, portugués e inglés). Luego amplié la revisión, con esos mismos criterios, a las revistas publicadas por Sage Publications. Desde ahí, proseguí en toda línea que me resultó prometedora (referencias que aparecían en algún artículo, artículos sobre temas "similares", otros trabajos de cierto autor, etc.) hasta donde el tiempo me lo permitió. De todo lo ahí encontrado, me permití seleccionar y organizar, con toda libertad, las ideas que finalmente aquí presento.

3. El sentido en el que estoy refiriéndome aquí a la investigación iberoamericana es el de la realizada por investigadores de habla hispana y portuguesa tanto en América Latina como en España y Portugal.

4. Que, en comparación con otras comunidades lingüísticas, no es una proporción fácil de ignorar.

5. Preocupación que, por supuesto, no es en modo alguno exclusiva de la investigación cualitativa iberoamericana en salud, sino que la comparten con los investigadores cualitativos que se ocupan de los más diversos temas en todas partes del mundo (Cornejo y Salas, 2011; Castillo, 2011).

6. Una cuestión, por cierto, que ha sido pensada y planteada desde tiempo atrás por importantes autores como Minayo (1993), Conde \& Pérez (1995) o Castro \& Bronfman (1999), entre otros.

\section{RESÚMENES}

Después de una atenta reflexión sobre su propia experiencia de investigación, la autora efectuó una amplia revisión de los reportes publicados en los años recientes por diversos investigadores cualitativos. A partir de ahí identificó tres grandes vertientes de las que parecen emerger buena parte de los desafíos que enfrentan quienes se ocupan de la problemática de salud desde estas perspectivas en Iberoamérica. En primer lugar, menciona los que emergen de la existencia misma de esta forma de generación de conocimiento. En segundo término, se refiere a los desafíos 
epistémico-metodológicos. Y por último, a los desafíos práctico-éticos. En un inicio, la imagen evocada para intentar representar la confluencia de estas tres vertientes fue la de un nudo Borromeo. Pero al final del recorrido, el intrincado entretejido de los componentes identificados la llevó a sugerir, más bien, la imagen de un nudo gordiano. El deseo de la autora con esta comunicación es convocar a quienes forman parte de esta comunidad de investigadores a mantener abierto el diálogo sobre las complejas implicaciones de esta modalidad de indagación.

Depois de uma reflexão cuidadosa sobre a sua própria experiência de investigação, a autora realizou uma extensa revisão dos relatórios de vários pesquisadores qualitativos nos últimos anos. Identifica três fontes principais dos desafios enfrentados pelos pesquisadores qualitativos que se concentram em questões de saúde nos países ibero-americanos. Menciona primeiramente os suscitados pela existência desse tipo de geração de conhecimento. Em segundo lugar, refere os desafios epistémico-metodológicas. E, finalmente, considera os desafios prático-éticos. Num momento inicial, a imagem evocada para tentar representar a confluência desses três domínios foi um nó borromeano. Contudo, no final do trajeto, a forma complexa em que estes componentes estão interligados levou-a a sugerir, em vez disso, a imagem de um nó górdio. O desejo da autora com esta comunicação é convidar a comunidade de pesquisadores a continuar com o diálogo sobre as implicações complexas deste tipo de investigação.

After a careful reflection on the insights obtained from her own research experience, the author conducted an extensive review of the reports of several qualitative researchers in recent years. She identifies three main sources of the challenges faced by Ibero-American qualitative researchers who focus on health issues. First, she mentions those posed by the very existence of this kind of knowledge generation. Second, she refers to the epistemic-methodological challenges. And finally, she considers the practical-ethical challenges. At first, the image evoked to try to represent the confluence of these three watersheds was a Borromean knot. But at the end of the journey, the intricate way in which these components are interwoven led her to suggest, rather, the image of a Gordian knot. The autor's desire with this communication is to invite this community of researchers to continue with the dialog about the complex implications of this kind of inquiry.

\section{ÍNDICE}

Keywords: qualitative health research, theoretical and methodological challenges, ethical and practical challenges

Palavras-chave: pesquisa qualitativa em saúde, desafios teórico-metodológicos, desafios éticos e práticos

Palabras claves: investigación cualitativa en salud, desafíos, problemas teórico-metodológicos, problemas ético-prácticos

\section{AUTOR}

\section{CAROLINA MARTÍNEZ-SALGADO}

Departamento de Atención a la Salud, Universidad Autónoma Metropolitana (Xochimilco), México (cmartine@correo.xoc.uam.mx) 